Pacific Journal of Mathematics

A CLASS OF ISOTROPIC COVARIANCE FUNCTIONS 


\section{A CLASS OF ISOTROPIC COVARIANCE FUNCTIONS}

\section{Yashaswini MitTal}

Let $\{f(x), x \geqq 0\}$ be nonnegative such that $\int_{0}^{\infty} f(x) d x=1$. Define $g(x)=f\left(|x| / s_{|x|, \pi}\right)$ for $x \in \mathbf{R}_{n}$. The $n$-dimensional Euclidean space is denoted by $\mathbf{R}_{n},|x|$ is the length of the vector $x \in \mathbf{R}_{n}$ and $S_{r, \pi}=$ surface area of the $n$-dimensional sphere with radius $r$. Let $W(d y)$ be the $(n+1)$-dimensional Gaussian white noise, i.e., for any Borel sets $B$ and $C$ in $\mathbf{R}_{n+1}, W(B)$ and $W(C)$ are mean zero Gaussian variables with variance of $W(B)=$ volume of $B$, and $E(W(B) W(C))=0$ if and only if $B \cap C=\varnothing$. Construct the sets $A_{t}$ in $\mathbf{R}_{n+1}$ as $A_{t}=$ $\left\{\left(x_{1} \cdots x_{n}, z\right) \in \mathbf{R}_{n} \times[0, \infty) \mid g(x+t)>z\right\}$. Define an $n$-dimensional isotropic Gaussian field as $X(t)=\int_{A_{t}} W(d y) ; t \in \mathbf{R}_{n}$. $X(t)$ has mean zero and variance one. In addition, if it is assumed that $f(x) / x^{n-1}$ is nonincreasing, then the covariance function of $x(t)$ can be computed to be $r(t)=$ $(2 / c) \int_{t / 2}^{\infty}\left(\int_{0}^{\theta} \sin ^{n-2} \alpha d \alpha\right) f(x) d x$, where $|t|=t, c=\int_{0}^{\pi} \sin ^{n-2} \alpha d \alpha$ and $\theta=\operatorname{arcos}(t / 2 x)$. Let $V_{n}$ denote the class of covariance functions $r(t)$ in $\mathbf{R}_{n}$. Characterizing properties of the class $V_{n}$ are studied for the odd and even dimensional spaces.

The class $V_{2}$ is the same as the one considered by Hájek and Zubrzycki. Some examples and the iterative properties of $V_{n}$ are also considered. The classical Pólya's criterion for characteristic functions is a special case of Theorem 1.

A collection of real or complex valued random variables $Z(t)$, where $t$ ranges over a $n$-dimensional Euclidean space $\mathbf{R}_{n}$ is called a "random field". Assume throughout that $E|Z(t)|^{2}$ is finite and that

$$
E|Z(t+\delta)-Z(t)|^{2} \rightarrow 0 \quad \text { as } \quad \delta \rightarrow 0 .
$$

Let $Z^{*}(t)$ denote the complex conjugate of $Z(t)$. The functions $E Z(t)=$ $m(t)$ and $K\left(t_{1}, t_{2}\right)=E\left(Z\left(t_{1}\right) Z^{*}\left(t_{2}\right)\right)-m\left(t_{1}\right) m^{*}\left(t_{2}\right)$ are called the "mean" and the "covariance" functions of $Z(t)$ respectively.

The class of covariance functions $K\left(K_{n}\right)$ is the same as that of the nonnegative definite functions in $\mathbf{R}_{n}$. The random field $Z(t)$ is called "isotropic" if $K\left(t_{1}, t_{2}\right)$ depends only on the length $\left|t_{1}-t_{2}\right|$ of the difference $t_{1}-t_{2}$. The Bochner-Khinchin theorem for nonnegative definite functions gives the spectral representation for isotropic covariance functions in $\mathbf{R}_{n}$. This leads to the following theorem (see [3] p. 39). 
THEOREM. For $R(t)$ to be the covariance function of an isotropic $n$-dimensional random field satisfying (0.1), it is necessary and sufficient that

$$
R(t)=2^{(n-2) / 2} \Gamma\left(\frac{n}{2}\right) \int_{0}^{\infty} \frac{J_{(n-2) / 2}(\lambda t)}{(\lambda t)^{(n-2) / 2}} d G(\lambda)
$$

where $G(\lambda)$ is bounded, nondecreasing function such that $G(0)=0$; $G(\infty)=R(0)$ and $J_{m}(x)$ is the Bessel function of the first kind of order $m$. Namely,

$$
J_{m}(x)=\sum_{K=0}^{\infty}(-1)^{K} \frac{(x / 2)^{2 K+m}}{K ! \Gamma(K+m+1)} .
$$

This paper considers a subclass $V_{n}$ of the isotropic covariance functions in $\mathbf{R}_{n}$. The classes $V_{n}$ arise naturally as the covariance functions of the integrated $(n+1)$-dimensional Gaussian white noise. Thus every covariance function given, comes with a representation of the associated isotropic Gaussian random field. Such representations can be used for simulation purposes.

Berman in his paper [1] gives a very interesting representation of covariance functions in $\mathbf{R}_{1}$ with absolutely continuous (abs. cont.) spectral distribution. The associated stationary Gaussian process is the integral of a two dimensional Gaussian white noise over appropriate sets. His techniques and results were used by Mittal and Ylvisaker [6] to generate a class of covariance functions in $\mathbf{R}_{1}$, that is similar to $V_{n}$.

The next section contains the statements of the main results. The proofs of these are achieved by a series of preliminary lemmas contained in $\S 2$. Section 3 proves the main results and the corollaries. The special case of $n=2$ is looked at in the last short section.

Notation. In the following, interpret $\sum_{i=1}^{0 \text { or-1 }}$ to be zero and the products $2 \cdot 4 \cdots(n-3)$ or $2 \cdot 4 \cdots(n-2)$ to be 1 if $n=3$ or $n=2$ respectively. A function $h(x)$ is $o(1, \infty)$ means $h(x) \rightarrow 0$ as $x \rightarrow \infty$ and it is $o(1,0)$ means $h(x) \rightarrow 0$ as $x \rightarrow 0 . \quad h^{(k)}$ is the $k$ th derivative of $h$ with $h^{(0)} \equiv h$.

1. Statements of results. Let $\{f(x), x \geqq 0\}$ be a density function, that is, $f(x) \geqq 0$ and $\int_{0}^{\infty} f(x) d x=1$. Then $g(x)=f(|x|) / S_{|x|, \pi}$ is a density function in $\mathbf{R}_{n}$ where $S_{u, \theta}=$ surface area of a sphere with radius $u$ intersected by a right circular cone of angle $\theta$ in $\mathbf{R}_{n}$. Let $W(d y)$ be the $(n+1)$-dimensional Gaussian white noise, that is, for any Borel sets $B$ and $C$ in $\mathbf{R}_{n+1}, W(B)$ and $W(C)$ are mean zero Gaussian variables with 
variance of $W(B)=$ volume of $B$ and $E(W(B) W(C))=$ volume of $(B \cap C)$. Define the sets $A_{t}$ as $\left\{\left(x_{1} \cdots x_{n}, z\right) \in \mathbf{R}_{n} \times[0, \infty) \mid g(x+t)>z\right\}$ where $x=\left(x_{1}, \cdots, x_{n}\right)$ and $t \in \mathbf{R}_{n}$. Define

$$
X(t)=\int_{A_{t}} W(d y)
$$

$X(t)$ is an isotropic Gaussian field in $\mathbf{R}_{n}$. In addition, assume that $f(x) / x^{n-1}$ is nonincreasing or that $g(x)$ is nonincreasing in $|x|$. The covariance function of $X(t)$ can be computed as follows. Let $|t|=t$.

$$
r(t)=E(X(0) X(t))=\text { volume of }\left(A_{t} \cap A_{0}\right) .
$$

Let $L$ be the $n$-dimensional plane orthogonal to the line joining $t$ to the origin. The volume of $A_{t} \cap A_{0}$ is symmetrically divided into halves by $L$. For computation of the volume on the side of $L$ that contains $t$, notice that it is bounded by $\mathbf{R}_{n}$ and the function $g$. Now $g$ takes constant values $f(|x|) S_{|x|, \pi}$ on $n$-dimensional spheres of radius $|x|$ centered at the origin. The surface area of the part of this sphere on the side of $L$ that contains $t$ is $S_{|x|, \theta}$ where $\theta=\operatorname{arcos}(t / 2|x|)$. Thus

$$
r(t)=2 \int_{t / 2}^{\infty} \frac{S_{|x|, \theta}}{S_{|x|, \pi}} f(|x|) d|x|
$$

Put $|x|=x$ and change to polar coordinates to evaluate $S_{x, \theta}$. Thus

$$
r(t)=\frac{2}{c} \int_{t / 2}^{\infty}\left(\int_{0}^{\theta} \sin ^{n-2} \alpha d \alpha f(x) d x\right)
$$

where $c=\int_{0}^{\pi} \sin ^{n-2} \alpha d \alpha$. The following theorem gives necessary and sufficient conditions for (1.2) to be an isotropic covariance function in odd dimensional spaces.

THEOREM 1. Let $n \geqq 3$ be an odd integer.

(A) For every density function $f$ on $[0, \infty)$ such that $f(x) / x^{n-1}$ is nonincreasing, $r(t)$ given by (1.2) is an isotropic covariance function in $\mathbf{R}_{n}$. It satisfies

(a) $r(t)$ is continuous, convex such that $r(t)=o(1, \infty)$ and $r(0)=1$.

(b) $r^{(k)}(t)$ are abs. cont. on $[\epsilon, \infty) \forall \epsilon>0$ and $t^{k} r^{(k)}(t)$ is $o(1, \infty)$ and $o(1,0)$ for $k=1,2, \cdots,(n-1) / 2$.

(c) $\frac{(-1)^{(n-1) / 2}}{t^{(n-1) / 2}}\left\{\sum_{i=1}^{(n-3) / 2} \frac{a_{i}^{(n+1) / 2}}{t^{i}} r^{(((n+1) / 2)-i)}(t)-r^{((n+1) / 2)}(t)\right\}$

is nonnegative and nonincreasing in $t$. 
(B) Let $r(t)$ satisfy (a), (b) and (c) above. Then $f(t)$ given by

$$
f(t / 2)=\frac{c t^{(n-1) / 2}(-1)^{(n-1) / 2}}{2 \cdot 4 \cdots(n-3)}\left\{\sum_{i=1}^{(n-3) / 2} \frac{a_{i}^{(n+1) / 2} r^{((n+1) / 2)-i)}(t)}{t^{i}}-r^{((n+1) / 2)}(t)\right\}
$$

is a density function on $[0, \infty)$ such that $f(x) / x^{n-1}$ is nonincreasing and $r(t)$ is an isotropic covariance function in $\mathbf{R}_{n}$ given by (1.2) for this $f$.

The constants $a_{i}^{k}$ are defined as

$$
\begin{aligned}
& a_{1}^{k}=\frac{(k-1)(k-2)}{2} ; \quad a_{k-2}^{k}=(-1)^{k-1} 1 \cdot 3 \cdot 5 \cdots(2 k-5), \quad \text { and } \\
& a_{i}^{k}=\frac{(k+i-2) !}{(2 i) !(k-i-2) !} a_{i}^{i+2}, \quad i=2,3, \cdots,(k-2)
\end{aligned}
$$

for $k=3,4, \cdots$ and $a_{i}^{k}=0$ otherwise.

The following theorem of Pólya can be viewed as a special case for $n=1$ of Theorem 1 with suitable changes in the notation.

THEOREM (Pólya). Let $r(t)$ be a real-valued and continuous function which is defined for all real $t$ and which satisfies the following conditions:

(i) $r(0)=1$,

(ii) $r(-t)=r(t)$,

(iii) $r(t)$ convex for $t>0$,

(iv) $\mathrm{lt}_{t \rightarrow \infty} r(t)=0$.

Then $r(t)$ is the characteristic function of an absolutely continuous distribution $F(x)$.

The next theorem gives characterization of the class $V_{n}$ of covariance functions given by (1.2) for $n=2 k, k=1,2, \cdots$.

THEOREM 2. Let $n \geqq 2$ be an even integer.

(A) For every density function $f$ on $[0, \infty)$ such that $f(x) / x^{n-1}$ is nonincreasing, $r(t)$ given by (1.2) is an isotropic covariance function in $\mathbf{R}_{n}$. It satisfies

(a) $\quad r(t)$ is continuous convex such that $r(t)=o(1, \infty)$ and $r(0)=1$.

(b) $r^{(k)}(t)$ is abs. cont. on $[\epsilon, \infty) \forall \epsilon>0$ and $r^{(k)}(t) t^{k}$ is $o(1, \infty)$ and $o(1,0)$ for $k=1,2, \cdots,(n / 2)$.

(c) $(-1)^{(n / 2)+1} \frac{d}{d t}\left\{t \int_{t}^{\infty} \frac{\beta(u)}{u^{n / 2} \sqrt{u^{2}-t^{2}}} d u\right\}$ 


$$
(-1)^{n / 2} \beta(u)=(-1)^{n / 2}\left(r^{(n / 2)}(u)-\sum_{i=1}^{(n / 2)-2} \frac{a_{i}^{n / 2}}{u^{i}} r^{((n / 2)-t)}(u)\right)
$$

is nonnegative.

(B) Let $r(t)$ satisfy (a), (b) and (c). Then $f(t)$ given by

$$
f(t / 2)=\frac{(-1)^{(n / 2)+1} \cdot 2 \cdot t^{n-1}}{2 \cdot 4 \cdots(n-2)} \cdot \frac{d}{d t}\left[t \int_{t}^{\infty} \frac{\beta(u)}{u^{n / 2} \sqrt{u^{2}-t^{2}}} d u\right]
$$

is a density function on $[0, \infty)$ such that $f(x) / x^{n-1}$ is nonincreasing and $r(t)$ is an isotropic covariance function in $R_{n}$ given by (1.2) for this $f$.

Corollary 1 gives a recursive property of the classes $V_{n}$ similar to that of $K_{n}$ given in Matérn ([5]; 2.3.12). Corollary 2 shows that $V_{n}$ is nonincreasing in $n$ and Corollary 3 proves that $V_{n}$ are closed under mixtures. The exact statements are as follows.

Corollary 1. If $r(t)$ is in the class $V_{n}$ for the associated density function $f$, then $\rho(t)$ defined as

$$
\rho(t)=\left(r(t)-\frac{t}{n-2} r^{\prime}(t)\right) \frac{n-2}{n-3} \cdot \frac{c_{n}}{c_{n-2}}
$$

belongs to the class $V_{n-2}$ for the same density function $f$ and $n \geqq 4$. $c_{n}=\int_{0}^{\pi} \sin ^{n-2} \alpha d \alpha$

COROLlaRy 2. (1) Let $r(t)$ be in $V_{n}, n \geqq 3$ and $f$ be the associated density function. Then $r \in V_{n-1}$ and the associated density function is given by

$$
g(t / 2)=2 \cdot \eta(n) \frac{t^{n-2}}{2^{n-2}} \int_{t / 2}^{\infty} \frac{f(x)}{x^{n-2} \sqrt{(2 x)^{2}-t^{2}}} d x
$$

where $\eta(n)=3 \cdot 5 \cdots(n-2) /(2 \cdot 4 \cdots(n-3))$ if $n$ is odd and $\eta(n)=$ $3 \cdot 5 \cdots(n-3) /(2 \cdot 4 \cdots(n-4))$ if $n$ is even.

(2) If $r$ is in $V_{n}$ for $n \geqq 4$ and $f$ is the associated density function then $r$ belongs to $V_{n-2}$ for the density function $g$ such that

$$
g(t / 2)=\frac{n-2}{2^{n-3}} t^{n-3} \int_{t / 2}^{\infty} \frac{f(x)}{x^{n-2}} d x .
$$

Corollary 3. Let $F$ be a distribution function with $F\left(0^{+}\right)=0$ and $r$ belongs to $V_{n}$ then $w(t)=\int_{0}^{\infty} r(t / a) d F(a)$ is a covariance function in $V_{n}$ for 
the density function $g(x)=\int_{0}^{\infty} f(x / a) \cdot a^{-1} d F(a) ; f$ being the density function of $r$.

The class $V_{2}$ is given particular attention, especially its relation with the class $\mathscr{F}$ of Hájek and Zubrzycki [2]. $\rho \in \mathscr{F}$ if and only if there exists a distribution function $G$ with $G\left(0^{+}\right)=0$ such that

$$
\rho(t)=\int_{0}^{\infty} \gamma\left(\frac{t}{a}\right) d G(a)
$$

where

Thus

$$
\begin{array}{rlrl}
\gamma(a) & =\frac{2}{\pi}\left[\operatorname{arcos} u-u\left(1-u^{2}\right)^{1 / 2}\right] & 0 \leqq & u \leqq 1 \\
& =0 & u>1 .
\end{array}
$$

$$
\begin{array}{rlrl}
\gamma^{\prime \prime}(u) & =\frac{4}{\pi} \cdot \frac{u}{\left(1-u^{2}\right)^{1 / 2}} & 0 \leqq & u<1 \\
& =0 & u & >1 .
\end{array}
$$

The following is Theorem 5.1 in [2].

THEOREM (Hájek, Zubrzycki). The correlation functions $\rho(t)$ given by (1.9) are characterized by the following properties

(i) $\quad \rho(t)$ is continuous, convex with $\rho(\infty)=0$

(ii) $\rho^{\prime}(t)$ is abs. cont.

(iii) $\int_{0}^{\infty}\left(1 / u^{2}\right) \gamma^{\prime \prime}(t / u) \rho^{\prime \prime}(u) d u$ is a nonincreasing function of $t$.

In the last section the following proposition is proved.

Proposition. (1) The covariance function $\gamma(u)$ given by $(1.10)$ belongs to $V_{2}$ for the associated density function $f(x)=8 x ; 0 \leqq x \leqq 1 / 2$.

(2) Every covariance function belonging to $V_{n}$ satisfies (iii) of the above theorem.

Thus $V_{2}=\mathscr{F}$.

2. Preliminary lemmas. The following lemmas will help to avoid repetitions in the proofs of the Theorems 1 and 2 .

LEMMA 1. Let $f(x)$ be a density function on $[0, \infty)$ such that $f(x) / x^{n-1}$ is nonincreasing. Define the functions for $K=1,2, \cdots,[n / 2]$ and $t>0$ 


$$
\begin{aligned}
I_{K}(t)= & \frac{(-1)^{K} t^{K-1}(n-3)(n-5) \cdots(n-2 K+1)}{c 4^{K-1}} \\
& \times \int_{t / 2}^{\infty}\left(1-\left(\frac{t}{2 x}\right)^{2}\right)^{(n-2 K-1) / 2} \frac{f(x)}{x^{2 K-1}} d x
\end{aligned}
$$

and $I_{K}(0)=l t_{t \rightarrow 0} I_{K}(t)$. The product $(n-3) \cdots(n-2 K+1)$ is interpreted to be 1 for either $K=1$ or $n=2,3$. Then $I_{K}(t)$ is bounded on $[\epsilon, \infty) \forall \epsilon>0$,

$$
\frac{d}{d t} I_{K}(t)=\frac{K-1}{t} I_{K}(t)+I_{K+1}(t)
$$

for $K=1,2, \cdots,[(n-2) / 2]$,

$$
\int_{t}^{\infty} I_{K}(u) d u=-I_{K-1}(u)-(K-2) \int_{t}^{\infty} \frac{I_{K-1}(u)}{u} d u
$$

for $K=2,3, \cdots,[n / 2]$ and

$$
t^{K} I_{K}(t) \text { is } o(1, \infty) \text { and } o(1,0)
$$

for $K=1,2, \cdots,[n / 2]$. Notice also that $I_{K}(t)$ has alternate signs for values of $K$, with $I_{1}(t)$ being negative.

Proof. The uniform convergence on $[\epsilon, \infty)$ of the integral in the R.H.S. of (2.1) is obvious for all $K$ such that $n-2 K-1>-1$. Suppose $n-2 K-1=-1$, i.e., $K=n / 2$, then the integral is

$$
\begin{aligned}
\int_{t / 2}^{\infty} \frac{f(x)}{\sqrt{x^{2}-(t / 2)^{2}}} \cdot \frac{1}{x^{n-2}} d x= & \int_{t / 2}^{(t / 2)+1} \frac{f(x)}{\sqrt{x-t / 2} \sqrt{x+t / 2}} \cdot \frac{1}{x^{n-2}} d x \\
& +\int_{(t / 2)+1}^{\infty} \frac{f(x)}{x^{n-2} \sqrt{x^{2}-(t / 2)^{2}}} d x .
\end{aligned}
$$

The second integral in the R.H.S. above is uniformly convergent since $n \geqq 2$. Now, $f$ cannot be unbounded on $[t / 2,(t / 2)+1]$ since $f(x) / x^{n-1}$ is nonincreasing. Hence the first part is uniformly convergent. By taking the derivatives under the integral sign, (2.2) follows.

$$
\begin{aligned}
\int_{t}^{\infty} I_{K}(u) d u= & \frac{(-1)^{K}(n-3)(n-5) \cdots(n-2 K+1)}{c 4^{K-1}} \\
& \times \int_{t / 2}^{\infty} \frac{f(x)}{x^{2 K-1}}\left(\int_{t}^{2 x} u^{K-1}\left(1-\left(\frac{u}{2 x}\right)^{2}\right)^{(n-2 K-1) / 2} d u\right) d x .
\end{aligned}
$$


The interchange of the order of the integration is justified by Fubini's theorem. The R.H.S. above is equal to

$$
\frac{4}{n-2 K+1} \int_{t / 2}^{\infty} \frac{f(x)}{x^{2 K-3}}\left[\int_{t}^{2 x} u^{K-2} d\left(\left(1-\left(\frac{u}{2 x}\right)^{2}\right)^{(n-2 K+1) / 2}\right)\right] d x
$$

apart from a constant provided $K \neq 1$ and $(n+1) / 2>K$. The above is

$$
\begin{aligned}
& \frac{4}{n-2 K+1}\left\{\int_{t / 2}^{\infty} \frac{f(x)}{x^{2 K-3}} t^{K-2}\left(1-\left(\frac{t}{2 x}\right)^{2}\right)^{(n-2 K+1) / 2} d x\right. \\
& \left.+(K-2) \int_{t}^{\infty} u^{K-3} \int_{u / 2}^{\infty}\left(1-\left(\frac{u}{2 x}\right)^{2}\right)^{(n-2 K+1) / 2} \frac{f(x)}{x^{2 K-3}} d x\right\} .
\end{aligned}
$$

The order of the integration in the second term is changed again by Fubini's theorem. Notice that for $K=2$, the second term is taken to be zero. Substituting values, $(2.3)$ is obtained.

Lastly, notice that $f(x) / x^{n-1}$ nonincreasing implies that

$$
0 \leqq \frac{1}{2} \frac{f(x)}{x^{n-2}} \leqq \int_{x / 2}^{x} \frac{f(u)}{u^{n-1}} d u \leqq\left(\frac{2}{x}\right)^{n-1} \int_{x / 2}^{x} f(u) d u
$$

and $x f(x)=o(1,0)$ and $o(1, \infty)$. For proving (2.4), we use the fact that $t^{K} I_{K}(t) \leqq \int_{t / 2}^{\infty} f(x) d x$ for all $t$ and $K \leqq(n-1) / 2$. Thus $t^{K} I_{K}(t)$ is $o(1, \infty)$. For $t$ small, choose $\lambda>t / 2$ so small that $x f(x) \leqq \epsilon \forall 0<x<\lambda$. Then

$$
\begin{aligned}
t^{K} I_{K}(t) \leqq & \epsilon t^{2 K-1} \int_{t / 2}^{\lambda}\left(1-\left(\frac{t}{2 x}\right)^{2}\right)^{(n-2 K-1) / 2} \frac{1}{x^{2 K}} d x \\
& +t^{2 K-1} \int_{\lambda}^{\infty}\left(1-\left(\frac{t}{2 x}\right)^{2}\right)^{(n-2 K-1) / 2} \frac{f(x)}{x^{2 K-1}} d x \\
\leqq & t^{2 K-1} \int_{t / 2}^{\lambda} \frac{d x}{x^{2 K-1} \sqrt{x^{2}-(t / 2)^{2}}}+\frac{t^{2 K-1}}{\lambda^{2 K-2} \sqrt{\lambda^{2}-(t / 2)^{2}}} \int_{\lambda}^{\infty} f(x) d x .
\end{aligned}
$$

Substituting $x=(t / 2) \sec \theta$ in the first integral in the R.H.S. above $t^{K} I_{K}(t)=o(1,0)$.

LEMmA 2. Let $f$ be a density function on $[0, \infty)$ such that $f(x) / x^{n-1}$ is nonincreasing and

$$
r(t)=\frac{2}{c} \int_{t / 2}^{\infty}\left(\int_{0}^{\theta} \sin ^{n-2} \alpha d \alpha\right) f(x) d x
$$

where $c$ and $\theta$ are defined at (1.2). Then 


$$
r^{(K)}(t)=\sum_{i=1}^{K-2} \frac{a_{i}^{K}}{t^{i}} r^{(K-t)}(t)+I_{K}(t)
$$

for $K=1,2, \cdots,[n / 2]$. The constants $a_{i}^{K}$ satisfy the recursive relations

(i) $a_{1}^{K+1}=a_{1}^{K}+K-1$

(ii) $a_{i}^{K+1}=a_{i}^{K}-(K+i-2) a_{i-1}^{K}$ for $i=2,3, \cdots,(K-2)$

(iii) $a_{K-1}^{K+1}=-(2 K-3) a_{K-2}^{K}$

for $K=3,4, \cdots,[n / 2]$ and $a_{1}^{1}=a_{1}^{2}=0 ; a_{1}^{3}=1$.

Proof. $f(x) / x^{n-1}$ nonincreasing implies that $f(x)$ is bounded on $[t / 2 ;(t / 2)+1]$. Hence the integral in the R.H.S. of $(2.5)$ is uniformly convergent for $K=1,2, \cdots,[n / 2]$. Differentiating under the integral sign would verify (2.6). Thus

$$
r^{(K+1)}(t)=\sum_{i=1}^{K-2}\left(\frac{a_{i}^{K} r^{(K-t+1)}(t)}{t^{1}}-\frac{i a_{i}^{K} r^{(K-t)}(t)}{t^{i+1}}\right)+\frac{K-1}{t} I_{K}(t)+I_{K+1}(t) .
$$

The constants $a_{i}^{K+1}$ for $i=1,2, \cdots,(K-1)$ are defined such that the R.H.S. above is

$$
\sum_{i=1}^{K-1} \frac{a_{i}^{K+1}}{t^{t}} r^{(K+1-t)}(t)+I_{K+1}(t)
$$

Some algebra will give (2.7).

Lemma 3. The constants $a_{i}^{K}$ of Lemma 2 are given by

(i) $\quad a_{1}^{K}=\frac{(K-1)(K-2)}{2}$

(2.8)

$$
\begin{aligned}
& \text { (ii) } a_{K-2}^{K}=(-1)^{K-1} 1 \cdot 3 \cdot 5 \cdots(2 K-5)=-a_{K-3}^{K} \\
& \text { (iii) } a_{\imath}^{K}=\frac{(K+i-2) !}{(2 i) !(K-i-2) !} a_{\imath}^{i+2} \text { for } i=2,3, \cdots,(K-2)
\end{aligned}
$$

for $K=3,4, \cdots$.

Proof. Direct computations give $a_{1}^{1}=a_{1}^{2}=0$ and $a_{1}^{3}=1$. Using (2.7) (i) and (iii), (2.8) (i) and (ii) are obtained. Now first (2.8) (iii) for $i=2$ is verified, that is,

$$
a_{2}^{K}=\left(\begin{array}{c}
K \\
4
\end{array}\right) a_{2}^{4}=(-1) \frac{K(K-1)(K-2)(K-3)}{2 \cdot 4} .
$$

Since $a_{2}^{4}=-3$. By (2.7) (iii), 


$$
\begin{aligned}
a_{2}^{K} & =a_{2}^{K-1}-(K-1) a_{1}^{K-1} \\
& =a_{2}^{K-2}-(K-2) a_{1}^{K-2}-(K-1) a_{1}^{K-1} \\
& \vdots \\
& =-3 a_{1}^{3}-4 a_{1}^{4}-\cdots-(K-1) a_{1}^{K-1} .
\end{aligned}
$$

Substituting the values for $a_{1}^{j}$, the R.H.S. becomes

$$
\begin{aligned}
-1 / 2 \sum_{l=1}^{K-3} l(l+1)(l+2) & \\
=-1 / 2\left\{\frac{(K-3)^{2}(K-2)^{2}}{4}+\frac{3(K-3)(K-2)(2 K-5)}{6}\right. & \left.+2 \frac{(K-3)(K-2)}{2}\right\} .
\end{aligned}
$$

Simplifying, (2.9) is verified. Now, assume that $(2.8$, iii) is true for $i=2,3, \cdots,(j-1)$. To show it for $j$, i.e.,

$$
a_{j}^{K}=\left(\begin{array}{c}
K+j-2 \\
2 j
\end{array}\right) a_{j}^{j+2}
$$

By (2.7 ii)

$$
\begin{aligned}
a_{j}^{K} & =a_{j}^{K-1}-(K-1+j-2) a_{j-1}^{K-1} \\
& =a_{j}^{K-2}-(K+j-4) a_{j-1}^{K-2}-(K+j-3) a_{j-1}^{K-1} . \\
& \vdots \\
& =-(2 j-1) a_{j-1}^{j+1}-2 j a_{j-1}^{j+2}-\cdots-(K+j-3) a_{j-1}^{K-1} .
\end{aligned}
$$

Using (2.8 iii) for $i=j-1$,

$$
\begin{aligned}
a_{j}^{K} & =-\left\{\sum_{l=0}^{K-j-2}(2 j+l-1) \frac{(2 j+l-2) !}{(2 j-2) ! l !}\right\} a_{j-1}^{j+1} \\
& =-(2 j-1) a_{j-1}^{j+1} \sum_{l=0}^{K-j-2}\left(\begin{array}{c}
2 j+l-1 \\
2 j-1
\end{array}\right) \\
& =\left(\begin{array}{c}
K+j-2 \\
2 j
\end{array}\right) a_{j}^{j+2} .
\end{aligned}
$$

(See e.g., [4], 0. 151). Simple substitution verifies $a_{K-2}^{K}=-a_{K-3}^{K}$.

Lemma 4. Let $r(t)$ be as in Lemma 2. Then (1) $r^{(K)}(t)$ are abs. cont. on $[\epsilon, \infty) \forall \epsilon>0$ for $K=0,1, \cdots,[n / 2]$ and

(2) $t^{K} r^{(K)}(t)$ is $o(1, \infty)$ and $o(1,0)$ for $K=1,2, \cdots,[n / 2]$. 
Proof.

$$
\int_{t}^{\infty} r(u) d u=-\frac{1}{c} \int_{t}^{\infty} \int_{u / 2}^{\infty}\left(1-\left(\frac{u}{2 x}\right)^{2}\right)^{(n-3) / 2} \frac{f(x)}{x} d x d u .
$$

Interchanging the order of integration by Fubini's theorem and substituting $u /(2 x)=\cos \alpha$, the R.H.S. above is seen to be equal to $-r(t)$. The abs. continuity of $r(t)$ follows by definition. Suppose $r^{(K)}(t)$ is abs. cont. for $K=0,1, \cdots,(j-1)$. Then $r^{(j)}(t)$ will be if $r^{(j+1)}(t)$ exists and is integrable. Looking at (2.6) for $K=j+1$, it is true if $I_{j+1}(t)$ is integrable. Lemma $1,(2.3)$ gives the result for $j+1 \leqq[n / 2]$. If $n$ is odd then $[n / 2]=(n-1) / 2$ and

$$
\begin{aligned}
r^{(n-1) / 2)}(t)= & \sum_{i=1}^{(n-5) / 2} \frac{a_{t}^{(n-1) / 2}}{t^{i}} r^{(n-1-2 t) / 2}(t) \\
& +\frac{(-1)^{K} t^{(n-3) / 2}(n-3)(n-5) \cdots 2}{c 4^{(n-3) / 2}} \int_{t / 2}^{\infty} \frac{f(x)}{x^{n-2}} d x
\end{aligned}
$$

The first part in the R.H.S. above involves $r^{(K)}(t)$ for $K=$ $1,2, \cdots,(n-3) / 2$ and the second part is abs. cont. by definition. If $n$ is even then $[n / 2]=n / 2$. By (2.6) for $K=n / 2$,

$$
\begin{aligned}
r^{(n / 2)}(t)= & \sum_{i=1}^{(n / 2)-2} \frac{a_{i}^{n / 2}}{t^{i}} r^{(n / 2)-i)}(t)+\frac{(-1)^{n / 2} \cdot 2 \cdot 4 \cdots(n-2)}{\pi 2^{n-2}} \\
& \cdot t^{(n / 2)-1} \int_{t / 2}^{\infty} \frac{f(x)}{x^{n-2} \sqrt{x^{2}-(t / 2)^{2}}} d x
\end{aligned}
$$

and it only needs to be shown that

$$
h(t)=t^{(n / 2)-1} \int_{t / 2}^{\infty} \frac{f(x)}{x^{n-2} \sqrt{x^{2}-(t / 2)^{2}}} d x
$$

is abs. cont. Define

$$
h^{\prime}(t)=\left(\frac{n}{2}-1\right) t^{(n / 2)-2} \int_{t / 2}^{\infty} \frac{f(x)}{\sqrt{x^{2}-(t / 2)^{2}}} d x+\frac{t^{n / 2}}{4} \int_{t / 2}^{\infty} \frac{1}{\sqrt{x^{2}-(t / 2)^{2}}} d\left(\frac{f(x)}{x^{n-1}}\right) .
$$

It will be shown that $\int_{t}^{\infty} h^{\prime}(u) d u=-h(t)$ for all $t>0$. Consider first

$$
\begin{aligned}
\frac{1}{4} \int_{t}^{\infty} u^{n / 2} \int_{u / 2}^{\infty} \frac{1}{\sqrt{x^{2}-(u / 2)^{2}}} d\left(\frac{f(x)}{x^{n-1}}\right) & d u \\
& =\frac{1}{4} \int_{t / 2}^{\infty} d\left(\frac{f(x)}{x^{n-1}}\right) \int_{t}^{2 x} \frac{u^{n / 2}}{\sqrt{x^{2}-(u / 2)^{2}}} d u
\end{aligned}
$$


by Fubini's theorem. The R.H.S. above is equal to

$$
\begin{aligned}
& \frac{1}{4} \int_{t / 2}^{\infty} d\left(\frac{f(x)}{x^{n-1}}\right) \int_{t}^{2 x} u^{(n / 2)-1} d\left(-4 \sqrt{x^{2}-(u / 2)^{2}}\right) \\
& =\int_{t / 2}^{\infty} d\left(\frac{f(x)}{x^{n-1}}\right)\left\{t^{(n / 2)-1} \sqrt{x^{2}-(t / 2)^{2}}+\left(\frac{n}{2}-1\right) \int_{t}^{2 x} u^{(n / 2)-2} \sqrt{x^{2}-(u / 2)^{2}} d u\right\} \\
& =t^{(n / 2)-1} \int_{t / 2}^{\infty} \sqrt{x^{2}-(t / 2)^{2}} d\left(\frac{f(x)}{x^{n-1}}\right) \\
& \quad+\left(\frac{n}{2}-1\right) \int_{t}^{\infty} u^{(n / 2)-2} \int_{u / 2}^{\infty} \sqrt{x^{2}-(u / 2)^{2}} d\left(\frac{f(x)}{x^{n-1}}\right) d u
\end{aligned}
$$

using Fubini's theorem again. (If $n=2$, the second term above is taken to be zero.) Finally, integrating by parts and noticing $x f(x)=o(1, \infty)$, the result is obtained.

Now, $r^{\prime}(t)=I_{1}(t)$ and (2) follows for $K=1$ by (2.4). Suppose (2) holds for $K=1,2, \cdots,(j-1)$. Then

$$
r^{(j)}(t)=\sum_{i=1}^{j-2} \frac{a_{i}^{j}}{t^{i}} r^{(j-i)}(t)+I_{j}(t)
$$

and (2) follows for $K=j$ by the above assumption and (2.4) provided $j \leqq[n / 2]$.

Lemma 5. Let $r(t)$ be as in Lemma 2. Then

$$
\int_{0}^{\infty} u^{K-1}\left\{\sum_{i=1}^{K-2} \frac{a_{i}^{K}}{u^{i}} r^{(K-i)}(u)-r^{(K)}(u)\right\} d u=-a_{K-1}^{K+1}
$$

for $K=2,3, \cdots,[(n+1) / 2] . \quad$ The constants $a_{i}^{K}$ are given in Lemma 2 and 3.

Proof. Using Lemma 3, first it will be verified that

$$
(K-i-1) a_{i}^{K+1}=(K+i-1) a_{i}^{K}
$$

for $i=1,2, \cdots,(K-2)$ and $K=2,3, \cdots,[(n+1) / 2]$. When $i=1,(2.12)$ states that $(K-2) a_{1}^{K+1}=K a_{1}^{K}$, which is true in view of $(2.7 \mathrm{i})$. By (2.8 iii), (2.12) becomes

$$
(K-i-1) \frac{(K+1+i-2) !}{(2 i) !(K-i-1) !}=(K+i-1) \frac{(K+i-2) !}{(2 i) !(K-i-2) !}
$$

for $i=2,3, \cdots,(K-2)$, which is an identity. 
Write $S_{j}(u)=\sum_{i=j}^{K-2} a_{i}^{K} u^{K-1-i} r^{(K-i)}(u)$. Then the L.H.S. in (2.11) is

$$
\begin{aligned}
\int_{0}^{\infty} u^{K-1} r^{(K)}(u) d u & +\int_{0}^{\infty} S_{1}(u) d u \\
& =\left(a_{1}^{K}+K-1\right) \int_{0}^{\infty} r^{(K-1)}(u) u^{K-2} d u+\int_{0}^{\infty} S_{2}(u) d u .
\end{aligned}
$$

Notice $r^{(K)}(u)$ are abs. cont. and $t^{K} r^{(K)}(u)=o(1, \infty)$ and $o(1,0)$ by part (2) of the last lemma for $K=0,1, \cdots,[(n-1) / 2]$. Successive integrations and use of (2.12) would evaluate the above to be

$$
a_{K-2}^{K+1} \int_{0}^{\infty} r^{(2)}(u) \cdot u d u=a_{K-2}^{K+1}=-a_{K-1}^{K+1}
$$

Hence the result.

3. Proof of the theorems and related results. The functions being considered are of the type

$$
r(t)=\frac{2}{c} \int_{t / 2}^{\infty}\left(\int_{0}^{\theta} \sin ^{n-2} \alpha d \alpha\right) f(x) d x
$$

where $\theta=\operatorname{arcos}(t / 2 x)$ and $c=\int_{0}^{\pi} \sin ^{n-2} \alpha d \alpha$.

Proof of Theorem 1. Note that $n \geqq 3$ is an odd integer.

Part A. $f(x)$ is a density function on $[0, \infty)$ such that $f(x) / x^{n-1}$ is nonincreasing and $x f(x)=o(1,0)$. (a), (b) and (c) in the statement of Theorem 1 from the last section must be proved.

Since $n \geqq 3$, the first two derivatives always exist, the first is negative and the second positive on $[\epsilon, \infty) \forall \epsilon>0$. Also $n$ odd implies $[(n-1) / 2]=$ $(n-1) / 2$. Lemma 4 and the following will show (b). Differentiating (2.6) for $K=(n-1) / 2$,

$$
\begin{aligned}
r^{((n+1) / 2)}(t)= & \sum_{i=1}^{(n-3) / 2} \frac{a_{i}^{(n+1) / 2} r^{(n+1-2 i) / 2}(t)}{t^{i}} \\
& -\left(\frac{(-1)^{(n-1) / 2} t^{(n-3) / 2}(n-3)(n-5) \cdots 4 \cdot 2}{c 4^{(n-3) / 2}}\right. \\
& \left.\times \frac{f(t / 2)}{(t / 2)^{n-2}} \cdot \frac{1}{2}\right) .
\end{aligned}
$$


Thus $t^{(n+1) / 2} r^{((n+1) / 2)}(t)$ is $o(1,0)$ if $r^{(n+1-2 i) / 2}(t) t^{(n+1-2 i) / 2}$ are for $i=$ $1,2, \cdots,(n-3) / 2$ and $t f(t)=o(1,0)$.

Lastly, the fact that the transform $r(t)$ is invertible is obvious from (3.2). It is equivalent to

$$
f(t / 2)=\frac{c t^{(n-1) / 2}(-1)^{(n-1) / 2}}{2 \cdot 4 \cdots(n-3)}\left\{\sum_{i=1}^{(n-3) / 2} \frac{a_{i}^{(n+1) / 2} r^{(n+1-2 i) / 2}(t)}{t^{i}}-r^{((n+1) / 2)}(t)\right\} .
$$

The quantity in (c) is in fact $f(t / 2) / t^{n-1}$ apart from a positive constant and hence nonnegative and nonincreasing by assumption.

Part B. $\quad r$ satisfies (a), (b) and (c). It needs to be shown that $r$ is given by (3.1) for $f$ given by (3.3) and $f$ is a density function on $[0, \infty)$ such that $f(x) / x^{n-1}$ is nonincreasing.

Since (3.3) is equivalent to (3.2) and $r^{(K)}(t)$ are abs. cont. for $X=0,1,2, \cdots,(n-1) / 2$, successive integrations will reduce (3.2) to (3.1). $f(x) / x^{n-1}$ nonincreasing follows easily from (c). It remains to be shown that $f$ is a density function.

$$
\begin{aligned}
\int_{0}^{\infty} f(t / 2) d t & =\frac{c(-1)^{(n-1) / 2}}{2 \cdot 4 \cdots(n-3)} \int_{0}^{\infty} u^{(n-1) / 2} \\
& \times\left\{\sum_{i=1}^{(n+1) / 2} \frac{a_{i}^{(n+1) / 2} r^{(n+1-2 i) / 2}(u)}{u^{i}}-r^{(n+1) / 2)}(u)\right\} d u \\
& =\frac{c(-1)^{(n+1) / 2}}{2 \cdot 4 \cdots(n-3)} a_{(n-1) / 2}^{(n+3) / 2} \quad \text { by }(2.11) \text { of Lemma } 5 \\
& =\frac{c(-1)^{n+1} 1 \cdot 3 \cdot 5 \cdots(n-2)}{2 \cdot 4 \cdots(n-3)} \quad \text { by Lemma 3 } \\
& =2
\end{aligned}
$$

since $n$ is odd and

$$
c=2 \int_{0} \sin ^{n-2} \alpha d \alpha=2 \cdot(2 \cdot 4 \cdots(n-3)) /(3 \cdot 5 \cdots(n-2)) .
$$

This completes the proof of Theorem 1.

Proof of Theorem 2. Now $n \geqq 2$ is an even integer.

Part A. $\quad r(t)$ is defined by (3.1) where $f$ is a density function with the usual assumptions. It will be shown that (a), (b) and (c) of the statement of Theorem 2 in the last section are satisfied. 
The function $r(t)$ is at least twice differentiable. $r^{\prime}(t)=I_{1}(t) \leqq 0$. If $n \geqq 4, r^{\prime \prime}(t)=I_{2}(t) \geqq 0$. Also if $n=2$

$$
r^{\prime \prime}(t)=\frac{1}{4 \pi} \int_{t / 2}^{\infty} \frac{t}{\sqrt{x^{2}-(t / 2)^{2}}} d\left(-\frac{f(x)}{x}\right) \geqq 0
$$

and $r$ is convex. Lemma 4 implies (b). To show (c), again it is verified that the transform $r(t)$ is invertible and the quantity in (c) is $f(t / 2) / t^{n-1}$ apart from a positive constant. (2.6) for $K=n / 2$ gives

(3.4) $r^{(n / 2)}(t)=\sum_{i=1}^{(n / 2)-2} \frac{a_{i}^{n / 2}}{t^{i}} r^{((n / 2)-i)}(t)$

$$
+\frac{(-1)^{n / 2} \cdot 2 \cdot 4 \cdots(n-2)}{\pi 2^{n-2}} t^{(n / 2)-1} \int_{t / 2}^{\infty} \frac{f(x)}{x^{n-1} \sqrt{1-(t / 2 x)^{2}}} d x .
$$

This is equivalent to

$$
\int_{u / 2}^{\infty} \frac{f(x)}{x^{n-2} \sqrt{x^{2}-(u / 2)^{2}}} d x=\frac{(-1)^{n / 2} \cdot 2^{n-2} \cdot \pi}{2 \cdot 4 \cdots(n-2)} \cdot \frac{\beta(u)}{u^{(n / 2)-1}}
$$

where

$$
\beta(u)=r^{(n / 2)}(u)-\sum_{i=1}^{(n / 2)-2} \frac{a_{i}^{n / 2}}{t^{i}} r^{((n / 2)-i)}(u)
$$

Notice that $(-1)^{n / 2} \beta(u) \geqq 0$. By Fubini's theorem

$$
\begin{aligned}
t \int_{t}^{\infty} \frac{1}{u^{2} \sqrt{1-(t / u)^{2}}} \int_{u / 2}^{\infty} \frac{f(x) / x^{n-1}}{\sqrt{1-\left(\frac{u}{2 x}\right)^{2}}} d x & \\
& =t \int_{t / 2}^{\infty} \frac{f(x) \cdot 2 x}{x^{n-1}} \int_{t}^{2 x} \frac{d u}{u \sqrt{u^{2}-t^{2}} \sqrt{(2 x)^{2}-u^{2}}} d x \\
& =\pi / 2 \int_{t / 2}^{\infty} \frac{f(x)}{x^{n-1}} d x .
\end{aligned}
$$

Thus

$$
\frac{2^{n-2} \cdot \pi(-1)^{n / 2}}{2 \cdot 4 \cdots(n-2)} \cdot t \int_{t}^{\infty} \frac{\beta(u)}{u^{(n / 2)+1} \sqrt{1-(t / u)^{2}}} d u=\pi / 2 \int_{t / 2}^{\infty} \frac{f(x)}{x^{n-1}} d x
$$

Taking derivatives and simplifying, 
$f(t / 2)=\frac{(-1)^{(n / 2)+1} 2 \cdot t^{n-1}}{2 \cdot 4 \cdots(n-2)} \frac{d}{d t}\left[t \int_{t}^{\infty} \frac{\left\{r^{(n / 2)}(u)-\sum_{i=1}^{(n / 2)-2} \frac{a_{i}^{n / 2}}{u^{i}} r^{((n / 2)-i)}(u)\right\}}{u^{n / 2} \sqrt{u^{2}-t^{2}}} d u\right]$

Part B. $\quad r$ satisfies (a), (b), (c) of the statement of Theorem 2 of last section. It needs to be shown that $r$ is given by (3.1) for $f$ given by (3.6) and $f$ is a density function on $[0, \infty)$. The fact that $f(x) / x^{n-1}$ is nonnegative and nonincreasing is obvious from (c).

First,

$$
\text { (3.7) } \begin{aligned}
\int_{0}^{\infty} f(t / 2) d t & =\frac{(-1)^{(n / 2)+1} \cdot 2}{2 \cdot 4 \cdots(n-2)} \int_{0}^{\infty} t^{n-1} d\left[t \int_{t}^{\infty} \frac{\beta(u)}{u^{n / 2} \sqrt{u^{2}-t^{2}}} d u\right] \\
& =-(n-1) \cdot \frac{(-1)^{(n / 2)+1} \cdot 2}{2 \cdot 4 \cdots(n-2)} \int_{0}^{\infty} t^{n-1} \int_{t}^{\infty} \frac{\beta(u)}{u^{n / 2} \sqrt{u^{2}-t^{2}}} d u d t .
\end{aligned}
$$

The last equality follows in view of (c) and using (b) to show that

$$
t^{n} \int_{t}^{\infty} \frac{\beta(u)}{u^{n / 2} \sqrt{u^{2}-t^{2}}} d u
$$

is $o(1,0)$ and $o(1, \infty)$. Since $(-1)^{n / 2} \beta(u)$ is nonnegative, by using Fubini's theorem in the above the integral in the R.H.S. becomes

$$
\begin{aligned}
-(n & -1) \int_{0}^{\infty} \frac{\beta(u)}{u^{n / 2}} \cdot\left(\int_{0}^{u} \frac{t^{n-1}}{\sqrt{u^{2}-t^{2}}} d t\right) d u \\
& =-(n-2) \cdot\left(\frac{n-4}{n-3} \cdots \cdot \frac{2}{3}\right) \int_{0}^{\infty} \beta(u) u^{(n / 2)-1} d u
\end{aligned}
$$

since

$$
\int_{0}^{u} \frac{t^{n-1}}{\sqrt{u^{2}-t^{2}}} d t=u^{n-1} \cdot \frac{n-2}{n-1} \cdots \cdot \frac{2}{3}
$$

(interpret the R.H.S. to be $u$ if $n=2$ ). By (2.12) of Lemma 5, $\int_{0}^{\infty} \beta(u) u^{(n / 2)-1} d u=a_{(n / 2)-1}^{(n / 2)+1}$. Substituting in (3.7), the total mass of $f$ is observed to be one.

To show that $r$ is given by (3.1), it is sufficient to establish the equality (3.4), in view of the abs. continuity of $r^{(K)}, K=0,1, \cdots, n / 2$. But (3.4) is equivalent to (3.5). Hence (3.5) will be established from (3.6). Write $W_{0}=(-1)^{(n / 2)+1} \cdot 2 \cdot 4 \cdots(n-2) / 2$. Thus

$$
W_{0} \int_{t}^{\infty} \frac{f(x / 2)}{x^{n-1}} d x=-t \int_{t}^{\infty} \frac{\beta(u)}{u^{n / 2} \sqrt{u^{2}-t^{2}}} d u
$$


Get $\beta(u)$ from (3.8) by the same inversion method used before.

$$
\begin{aligned}
t \int_{t}^{\infty} \frac{1}{u^{2} \sqrt{u^{2}-t^{2}}} \cdot u \int_{u}^{\infty} \frac{\beta(x)}{x^{n / 2} \sqrt{x^{2}-u^{2}}} & d x d u \\
& =t \int_{t}^{\infty} \frac{\beta(x)}{x^{n / 2}} \int_{t}^{x} \frac{d u}{u \sqrt{u^{2}-t^{2}} \sqrt{x^{2}-u^{2}}} d x \\
& =\frac{\pi}{2} \int_{t}^{\infty} \frac{\beta(x)}{x^{(n / 2)+1}} d x .
\end{aligned}
$$

Thus

$$
\begin{aligned}
\frac{\pi}{2} \int_{t}^{\infty} \frac{\beta(x)}{x^{(n / 2)+1}} d x & =-t W_{0} \int_{t}^{\infty} \frac{1}{u^{2} \sqrt{u^{2}-t^{2}}} \int_{u}^{\infty} \frac{f(x / 2)}{x^{n-1}} d x d u \\
& =-t W_{0} \int_{t}^{\infty} \frac{f(x / 2)}{x^{n-1}} \int_{t}^{x} \frac{d u}{u^{2} \sqrt{u^{2}-t^{2}}} \cdot d x \\
& =-W_{0} \int_{t}^{\infty} \frac{f(x / 2)}{x^{n}} \frac{\sqrt{x^{2}-t^{2}}}{t} d x .
\end{aligned}
$$

Taking derivatives on both sides and simplifying, follows. This completes the proof of Theorem 2 .

Proof of Corollary 1. For $r \in V_{n}$, it needs to be shown that

$$
\rho(t)=\left(r(t)-\frac{t}{n-2} r^{\prime}(t)\right) \frac{n-2}{n-3} \cdot \frac{c_{n}}{c_{n-2}}
$$

belongs to the class $V_{n-2}$ for all $n \geqq 4\left(c_{n}=\int_{0}^{\pi} \sin ^{n-2} \alpha d \alpha\right)$ and that $\rho$ and $r$ have the same associated density function.

$$
r(t)=\frac{2}{c_{n}} \int_{t / 2}^{\theta}\left(\int_{0}^{\theta} \sin ^{n-2} \alpha d \alpha\right) f(x) d x
$$

where $\theta=\operatorname{arcos}(t / 2 x)$. Thus

$$
r^{\prime}(t)=-\frac{1}{c} \int_{t / 2}^{\infty}\left(1-\left(\frac{t}{2 x}\right)^{2}\right)^{(n-3) / 2} \frac{f(x)}{x} d x
$$

Now

$$
\begin{aligned}
& \int_{0}^{\theta} \sin ^{n-2} \alpha d \alpha=\int_{0}^{\theta} \sin ^{n-3} \alpha \sin \alpha d \alpha \\
& \quad=-\cos \theta\left(1-\cos ^{2} \theta\right)^{(n-3) / 2}+(n-3) \int_{0}^{\theta} \sin ^{n-4} \alpha d \alpha-(n-3) \int_{0}^{\theta} \sin ^{n-2} \alpha d \alpha .
\end{aligned}
$$


Transferring the third term in the R.H.S. to L.H.S.,

$$
(n-2) \int_{0}^{\theta} \sin ^{n-2} \alpha d \alpha=(n-3) \int_{0}^{\theta} \sin ^{n-4} \alpha d \alpha-\frac{t}{2 x}\left(1-\left(\frac{t}{2 x}\right)^{2}\right)^{(n-3) / 2}
$$

Thus

$$
\begin{aligned}
r(t)= & \frac{2}{c_{n}(n-2)}\left\{(n-3) \int_{t / 2}^{\infty}\left(\int_{0}^{\theta} \sin ^{n-4} \alpha d \alpha\right) f(x) d x\right. \\
& \left.-\frac{t}{2} \int_{t / 2}^{\infty}\left(1-\left(\frac{t}{2 x}\right)^{2}\right)^{(n-3) / 2} \cdot \frac{f(x)}{x} d x\right\} .
\end{aligned}
$$

Substituting,

$$
\rho(t)=\frac{2}{c_{n-2}} \int_{t / 2}^{\infty}\left(\int_{0}^{\theta} \sin ^{n-4} \alpha d \alpha\right) f(x) d x .
$$

The result follows from the definition of the classes $V_{n}$.

Proof of Corollary 2. The objective is to show that $V_{n} \supseteq V_{n-1} \supseteq \cdots$. Let $r$ be given by (3.10). Suppose $n \geqq 3$ is odd; then by Theorem 1, part A, the conditions (a) and (b) of Theorem 2 will be satisfied by $r$, for $m=n-1$. It remains to be proved that

$$
(-1)^{(m+2) / 2} \frac{d}{d t}\left\{t \int_{t}^{\infty} \frac{\beta(u)}{u^{m / 2} \sqrt{u^{2}-t^{2}}} d u\right\}
$$

is nonnegative and nonincreasing where

$$
\beta(u)=r^{(m / 2)}(u)-\sum_{i=1}^{(m / 2)-2} \frac{a_{i}^{m / 2}}{u^{i}} r^{(m-2 i) / 2}(u)
$$

is such that $(-1)^{m / 2} \beta(u) \geqq 0 . \quad$ By (2.6) for $K=n-1$,

where

$$
\beta(u)=W_{1} u^{(n-3) / 2} \int_{u / 2}^{\infty} \frac{f(x)}{x^{n-2}} d x
$$

$$
W_{1}=\frac{(-1)^{(n-1) / 2} \cdot(n-3)(n-5) \cdots 2}{c 4^{(n-3) / 2}}
$$

Therefore

$$
\begin{aligned}
\int_{t}^{\infty} \frac{\beta(u)}{u^{m / 2} \sqrt{u^{2}-t^{2}}} d u & =W_{1} \int_{t}^{\infty} \frac{1}{u \sqrt{u^{2}-t^{2}}} \int_{u / 2}^{\infty} \frac{f(x)}{x^{n-2}} d x d u \\
& =W_{1} \int_{t / 2}^{\infty} \frac{f(x)}{x^{n-2}} \int_{t}^{2 x} \frac{d u}{u \sqrt{u^{2}-t^{2}}} d x \\
& =W_{1} \int_{t / 2}^{\infty} \frac{f(x)}{x^{n-2}} \operatorname{arcos} \frac{t}{2 x} d x
\end{aligned}
$$


The quantity in (3.11) is thus

$$
\frac{3 \cdot 5 \cdots(n-3)}{2^{n-2}} \int_{t / 2}^{\infty} \frac{f(x)}{x^{n-2} \sqrt{(2 x)^{2}-t^{2}}} d x
$$

The derivative under the integral sign in the last step is valid because of the uniform convergence of the integral in (3.12). Next it will be shown that (3.12) is nonincreasing in $t$. Let $\delta>0$,

$$
\begin{aligned}
\int_{t / 2}^{\infty} & \frac{f(x)}{x^{n-2} \sqrt{(2 x)^{2}-t^{2}}} d x-\int_{(t+\delta) / 2}^{\infty} \frac{f(x)}{x^{n-2} \sqrt{(2 x)^{2}-(t+\delta)^{2}}} d x \\
= & \int_{t / 2}^{(t+\delta) / 2} \frac{f(x)}{x^{n-2} \sqrt{(2 x)^{2}-t^{2}}} d x \\
& \quad-\int_{(t+\delta) / 2}^{\infty} \frac{f(x)}{x^{n-2}}\left\{\frac{1}{\sqrt{(2 x)^{2}-(t+\delta)^{2}}}-\frac{1}{\sqrt{(2 x)^{2}-t^{2}}}\right\} d x
\end{aligned}
$$

But

$$
\begin{aligned}
\int_{t / 2}^{(t+\delta) / 2} \frac{f(x)}{x^{n-2} \sqrt{(2 x)^{2}-t^{2}}} d x & =\int_{t / 2}^{(t+\delta) / 2} \frac{f(x)}{x^{n-1}} d\left(\frac{\sqrt{(2 x)^{2}-t^{2}}}{4}\right) \\
& \geqq \frac{f\left(\frac{t+\delta}{2}\right)}{\left(\frac{t+\delta}{2}\right)^{n-1}} \cdot \frac{1}{4} \cdot \sqrt{2 t \delta+\delta^{2}}
\end{aligned}
$$

Note that $f(x) / x^{n-1}$ is nonincreasing. Also

$$
\begin{aligned}
\int_{(t+\delta) / 2}^{\infty} & \frac{f(x)}{x^{n-2}}\left\{\frac{1}{\sqrt{(2 x)^{2}-(t+\delta)^{2}}}-\frac{1}{\sqrt{(2 x)^{2}-t^{2}}}\right\} d x \\
\leqq & \frac{\left(2 t \delta+\delta^{2}\right) \cdot f\left(\frac{t+\delta}{2}\right)}{\left(\frac{t+\delta}{2}\right)^{n-2} 2} \int_{(t+\delta) / 2}^{\infty} \frac{x}{\left((2 x)^{2}-(t+\delta)^{2}\right)^{3 / 2}} d x \\
= & \frac{\left(2 t \delta+\delta^{2}\right)^{1 / 2} f\left(\frac{t+\delta}{2}\right)}{8 \cdot\left(\frac{t+\delta}{2}\right)^{n-1}} .
\end{aligned}
$$

Substituting, (3.12) is nonincreasing. By part B of Theorem 2, $r$ must belong to $V_{n-1}$. The associated density function in $\mathbf{R}_{n-1}$ will be given by 


$$
\begin{aligned}
g(t / 2) & =\frac{(-1)^{m / 2+1} \cdot 2 \cdot t^{m-1}}{2 \cdot 4 \cdots(m-2)} \frac{d}{d t}\left\{t \int_{t}^{\infty} \frac{\beta(u)}{u^{m / 2} \sqrt{u^{2}-t^{2}}} d u\right\} \\
& =\frac{2 \cdot t^{n-2}}{2^{n-2}} \cdot \frac{3 \cdot 5 \cdots(n-2)}{2 \cdot 4 \cdots(n-3)} \int_{t / 2}^{\infty} \frac{f(x)}{x^{n-2} \sqrt{(2 x)^{2}-t^{2}}} d x .
\end{aligned}
$$

If $n$ is even, then $m=n-1$ is odd and by Theorem 2 part A, the conditions (a) and (b) of Theorem 1 are satisfied for $m$. It remains to verify that

$$
\frac{(-1)^{(m-1) / 2}}{t^{(m-1) / 2}}\left\{\sum_{i=1}^{(m-3) / 2} \frac{a_{i}^{(m+1) / 2}}{t^{i}} r^{(m+1-2 i) / 2}(t)-r^{((m+1) / 2)}(t)\right\}
$$

is nonnegative and nonincreasing. But by (2.6) for $K=n / 2$, the quantity in (3.13) is

$$
2 \cdot \frac{(-1)^{n}(n-3) \cdots 5 \cdot 3}{c 4^{(n / 2)-1}} \int_{t / 2}^{\infty} \frac{f(x)}{x^{n-2} \sqrt{(2 x)^{2}-t^{2}}} d x .
$$

The rest of the proof is similar to the first part.

Proof of Corollary 3. Let $F$ be a distribution function with $F\left(0^{+}\right)=$ 0 and let

$$
\gamma(t)=\int_{0}^{\infty}\left[\frac{2}{c} \int_{t / a}^{\infty}\left(\int_{0}^{\operatorname{arcos}(t / 2 x a)} \sin ^{n-2} \alpha d \alpha\right) f(x) d x\right] d F(a) .
$$

Making the transformation $x a=v$,

$$
\begin{aligned}
\gamma(t) & =\int_{0}^{\infty}\left[\frac{2}{c} \int_{t}^{\infty}\left(\int_{0}^{\operatorname{arcos}(t / 2 v)} \sin ^{n-2} \alpha d \alpha\right) f\left(\frac{v}{a}\right) \cdot \frac{1}{a} d v\right] d F(a) \\
& =\frac{2}{c} \int_{t}^{\infty}\left(\int_{0}^{\theta} \sin ^{n-2} \alpha d \alpha\right)\left(\int_{0}^{\infty} f\left(\frac{x}{a}\right) \frac{1}{a} d F(a)\right) d x
\end{aligned}
$$

where $\theta=\operatorname{arcos}(t / 2 x)$. Define $g(x)=\int_{0}^{\infty} f(x / a)(1 / a) d F(a)$. Then $g(x) \geqq 0$, and

$$
\int_{0}^{\infty} g(x) d x=\int_{0}^{\infty}\left(\int_{0}^{\infty} f\left(\frac{x}{a}\right) \cdot \frac{1}{a} d F(a)\right) d x=1 .
$$

Also

$$
\frac{g(x)}{x^{n-1}}=\int_{0}^{\infty} \frac{f(x / a)}{(x / a)^{n-1}} \cdot \frac{1}{a^{n}} d F(a)
$$

is nonincreasing in $x$. 
4. The two dimensional case. Let $\mathscr{F}$ be Hájek and Zubrzycki's class of planar covariance functions. The class is generated by the covariance function

$$
\begin{array}{rlrl}
\gamma(u) & =\frac{2}{\pi}\left[\operatorname{arcos} u-u\left(1-u^{2}\right)^{1 / 2}\right] & 0 \leqq & u \leqq 1 \\
& =0 & u & >1 .
\end{array}
$$

Proof of the Proposition. (1) $\gamma(u)$ satisfies the requirements of part $\mathrm{B}$ of Theorem 2. Hence $\gamma \in V_{2}$ and the associated density function $f$ is such that

$$
\begin{aligned}
f(x / 2) & =-\frac{8 x}{\pi} \frac{d}{d x}\left\{x \int_{x}^{1} \frac{\sqrt{1-u^{2}}}{u \sqrt{u^{2}-x^{2}}} d u\right\} & & 0 \leqq x \leqq 1 \\
& =-\frac{8 x}{\pi} \frac{d}{d x}\left\{x\left(\frac{\pi}{2 x}-\frac{\pi}{2}\right)\right\} & & 0 \leqq x \leqq 1 \\
& =4 x & & 0 \leqq x \leqq 1 .
\end{aligned}
$$

(2) $r \in V_{2}$, hence

$$
r^{\prime \prime}(t)=\frac{1}{4 \pi} \int_{t / 2}^{\infty} \frac{t}{\sqrt{x^{2}-(t / 2)^{2}}} d\left(-\frac{f(x)}{x}\right) .
$$

The proof will be complete if

$$
\int_{0}^{\infty} \frac{1}{u^{2}} \gamma^{\prime \prime}\left(\frac{t}{u}\right) r^{\prime \prime}(u) d u
$$

is a nonincreasing function of $t$, where $\gamma^{\prime \prime}(u)$ is given by (1.11). The quantity in (4.2) is

$$
\begin{aligned}
& \frac{4}{\pi} \cdot \frac{t}{4 \cdot \pi} \int_{t}^{\infty} \frac{d u}{u \sqrt{u^{2}-t^{2}}} \int_{u / 2}^{\infty} \frac{d(-f(x) / x)}{\sqrt{x^{2}-(u / 2)^{2}}} \\
& =\frac{t}{\pi^{2}} \int_{t / 2}^{\infty} d\left(-\frac{f(x)}{x}\right)\left(\int_{t}^{2 x} \frac{2 \cdot d u}{u \sqrt{u^{2}-t^{2}} \sqrt{(2 x)^{2}-t^{2}}}\right) d x \text { by Fubini's theorem } \\
& =\frac{1}{2 \pi} \int_{t / 2}^{\infty} \frac{1}{x} d\left(-\frac{f(x)}{x}\right)
\end{aligned}
$$

Note that

$$
d F(a)=\frac{1}{4}\left(f(a / 2)-(a / 2) f^{\prime}(a / 2)\right) .
$$


Hájek and Zubrzycki [2] show that the covariance functions $e^{-c t}$ and $(2 / \sqrt{2 \pi}) \int_{t}^{\infty} e^{-u^{2} / 2} d u$ belong to $\mathscr{F}$ and hence to $V_{2}$. They also have an example of an isotropic covariance function that does not belong to $\mathscr{F}$.

\section{REFERENCES}

1. S. M. Berman, A new characterization of characteristic functions of absolutely continuous distributions, To appear in Pacific J. Math. (1975).

2. T. Dalenius, J. Hájek and S. Zubrzycki, On plane sampling and related geometrical problems, Fourth Berkeley Symposium, I, (1961), 125-150.

3. I. Gikhman and A. Skorokhod, Introduction to the theory of random processes. W. B. Saunders Company, Philadelphia, (1969).

4. I. S. Gradshteyn and I. M. Ryzhik, Table of integrals, series and products. Academic Press, New York, (1965).

5. B. Matérn, Special variation, Medd. Statens Skogsforskningsistitut. Band 49, NR5, (1960).

6. Yash Mittal and Donald Ylvisaker, Strong laws for the maxima of stationary Gaussian processes, Ann. Prob. 4 No. 3, 357-372.

Received September 30, 1975. This research was partially supported by NSF Grant No. MPS 74-21416.

STANFORD UNIVERSITY 


\section{PACIFIC JOURNAL OF MATHEMATICS}

\section{EDITORS}

RICHARD ARENS (Managing Editor)

University of California

Los Angeles, California 90024

\author{
R. A. Beaumont \\ University of Washington \\ Seattle, Washington 98105
}

\section{J. DugunduI}

Department of Mathematics University of Southern California Los Angeles, California 90007

D. Gilbarg and J. Milgram

Stanford University

Stanford, California 94305

\section{ASSOCIATE EDITORS}

E. F. BECKENBACH
B. H. NeumanN

F. Wolp
K. YoshidA

\section{SUPPORTING INSTITUTIONS}

UNIVERSITY OF BRITISH COLUMBIA

CALIFORNIA INSTITUTE OF TECHNOLOGY

UNIVERSITY OF CALIFORNIA

MONTANA STATE UNIVERSITY

UNIVERSITY OF NEVADA

NEW MEXICO STATE UNIVERSITY

OREGON STATE UNIVERSITY

UNIVERSITY OF OREGON

OSAKA UNIVERSITY

\author{
UNIVERSITY OF SOUTHERN CALIFORNIA \\ STANFORD UNIVERSITY \\ UNIVERSITY OF HAWAII \\ UNIVERSITY OF TOKYO \\ UNIVERSITY OF UTAH \\ WASHINGTON STATE UNIVERSITY \\ UNIVERSITY OF WASHINGTON \\ AMERICAN MATHEMATICAL SOCIETY
}

The Supporting Institutions listed above contribute to the cost of publication of this Journal, but they are not owners or publishers and have no responsibility for its contents or policies.

Mathematical papers intended for publication in the Pacific Journal of Mathematics should be in typed form or offset-reproduced (not dittoed). double spaced with large margins. Underline Greek letters in red, German in green, and script in blue. The first paragraph or two must be capable of being used separately as a synopsis of the entire paper. Items of the biblography should not be cited there unless absolutely necessary, in which case they must be identified by author and Journal, rather than by item number. Manuscripts, in duplicate, may be sent to any one of the four editors. Please classify according to the scheme of Math. Reviews, Index to Vol. 39. All other communications should be addressed to the managing editor, or Elaine Barth, University of California, Los Angeles, California, 90024.

100 reprints are provided free for each article, only if page charges have been substantially paid. Additional copies may be obtained at cost in multiples of 50 .

The Pacific Journal of Mathematics is issued monthly as of January 1966. Regular subscription rate: $\$ 72.00$ a year (6 Vols., 12 issues). Special rate: $\$ 36.00$ a year to individual members of supporting institutions.

Subscriptions, orders for back numbers, and changes of address should be sent to Pacific Journal of Mathematics, 103 Highland Boulevard, Berkeley, California, 94708.

PUBLISHED BY PACIFIC JOURNAL OF MATHEMATICS, A NON-PROFIT CORPORATION Printed at Jerusalem Academic Press, POB 2390, Jerusalem, Israel.

Copyright (C) 1976 Pacific Journal of Mathematics All Rights Reserved 


\section{Pacific Journal of Mathematics}

\section{Vol. 64, No. $2 \quad$ June, 1976}

Richard Fairbanks Arnold and A. P. Morse, Plus and times............. 297

Edwin Ogilvie Buchman and F. A. Valentine, External visibility ......... 333

R. A. Czerwinski, Bonded quadratic division algebras.............. 341

William Richard Emerson, Averaging strongly subadditive set functions in unimodular amenable groups. II .........................

Lynn Harry Erbe, Existence of oscillatory solutions and asymptotic behavior for a class of third order linear differential equations ............. 369

Kenneth R. Goodearl, Power-cancellation of groups and modules........ 387

J. C. Hankins and Roy Martin Rakestraw, The extremal structure of locally compact convex sets ...................................

Burrell Washington Helton, The solution of a Stieltjes-Volterra integral

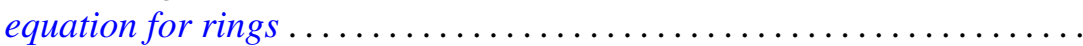

Frank Kwang-Ming Hwang and Shen Lin, Construction of 2-balanced

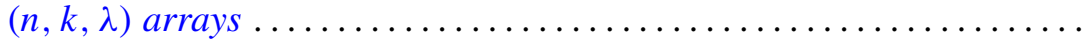

Wei-Eihn Kuan, Some results on normality of a graded ring ... 455

Dieter Landers and Lothar Rogge, Relations between convergence of series and convergence of sequences ......................... 465

Lawrence Louis Larmore and Robert David Rigdon, Enumerating immersions and embeddings of projective spaces ................

Douglas C. McMahon, On the role of an abelian phase group in relativized problems in topological dynamics..................

Robert Wilmer Miller, Finitely generated projective modules and TTF classes...

Yashaswini Deval Mittal, A class of isotropic covariance functions ...

Anthony G. Mucci, Another martingale convergence theorem ...

Joan Kathryn Plastiras, Quasitriangular operator algebras ...

John Robert Quine, Jr., The geometry of $p\left(S^{1}\right) \ldots \ldots \ldots$. 